\title{
Efeitos do alongamento na qualidade de vida e flexibilidade de mulheres jovens
}

\section{Effects of stretching on the quality of life and flexibility of young women}

\author{
Juliana Pracideli1; Cristina Maria Nunes Cabral ${ }^{2}$ \\ ${ }^{1}$ Fisioterapeuta, Bolsista PIBIC - UNIFIEO. Osasco, SP - Brasil. \\ ${ }^{2}$ Fisioterapeuta, Professora Doutora do Programa de Mestrado em Fisioterapia - UNICID, São Paulo, SP - Brasil. \\ Endereço para correspondência \\ Cristina Maria Nunes Cabral \\ R. Cesário Galeno 475, \\ 03071-000 - São Paulo - SP [Brasil] \\ ccabral@edu.unicid.br
}

\begin{abstract}
Resumo
Objetivo: Analisar o impacto do alongamento muscular sobre a qualidade de vida e flexibilidade de mulheres jovens. Métodos: Foram selecionadas 11 participantes, com baixos escores de qualidade de vida avaliada pelo SF-36, que realizaram um protocolo de alongamento muscular geral durante quatro semanas. A avaliação foi realizada antes, imediatamente após e um ano após o alongamento, utilizando o SF-36 e a medida da distância terceiro dedo-solo. Resultados: Houve diferença estatisticamente significante em quase todos os aspectos da qualidade de vida após um ano $(p<0,05)$, exceto no aspecto de capacidade funcional $(p=0,20)$ e estado geral de saúde $(\mathrm{p}=0,08)$. Na medida da distância terceiro dedo-solo também se observou diferença estatística importante entre as três avaliações $(p=0,02)$. Conclusão: Estes resultados sugerem que, após um período de tempo, os escores do SF-36 para avaliação da qualidade de vida diminuem, mas não retornam aos iniciais. Ainda, pode-se observar diminuição da amplitude de movimento após um ano.
\end{abstract}

Descritores: Amplitude de Movimento Articular; Qualidade de vida; Questionários; Seguimentos.

\begin{abstract}
Objective: To analyze the impact of muscular stretching on the quality of life and flexibility of young women. Methods: Eleven students who obtained low scores of quality of life in the SF-36 were selected, and performed a protocol of general muscular stretching during four weeks. The evaluation occurred before, immediately after and one year after the muscular stretching, using the SF-36 and measurement between the third fingertip-floor. Results: A significant difference was observed in almost all the aspects of the quality of life after one year $(p<0.05)$, except in the physical function $(p=0.20)$ and general health $(p=0.08)$. The third fingertip-floor distance measurement showed significant differences in the three evaluations $(p=0.02)$. Conclusion: These results suggest that after a period of time the SF-36 scores for assessing the quality of life diminish, but do not return to the initial values. Moreover, a reduction of the range of motion can be observed after one year.
\end{abstract}

Key words: Follow-up studies; Quality of life; Questionnaires; Range of motion, articular. 


\section{Introdução}

A qualidade de vida pode ser relacionada à saúde e entendida como a determinação do impacto social, físico e psicológico imposto por uma doença ${ }^{1}$. Assim, a percepção do estado de saúde e da qualidade de vida dos pacientes, bem como o impacto tanto da sua doença como de seu respectivo tratamento estão sendo amplamente reconhecidos como um tópico de pesquisa em estudos clínicos e epidemiológicos². É possível avaliar a qualidade de vida pela administração de questionários, os quais podem ser divididos em genéricos e específicos ${ }^{2,3,4,5}$. O SF-36 é um instrumento genérico de avaliação da qualidade de vida2, 6,7, autoadministrável, composto por 36 itens que englobam oito escalas, a saber: capacidade funcional, aspectos físicos, dor, estado geral de saúde, vitalidade, aspectos sociais, aspectos emocionais e saúde mental. Possui um escore final de zero a 100, em que zero corresponde a um pior estado geral de saúde; e 100, a um melhor estado geral de saúde $^{2,7,8,9,10}$.

Atualmente, percebe-se a existência de uma linguagem voltada à qualidade de vida associada à prática de atividade física, que vem ganhando espaço na prevenção, conservação e melhora dos padrões fisiológicos do ser humano ${ }^{11}$. A inatividade física é associada ao risco de diversas doenças e condições crônicas de saúde (entre elas, a obesidade e doenças cardiovasculares); e o papel do comportamento sedentário vem ganhando cada vez mais atenção na saúde pública ${ }^{12,13}$. O sedentarismo também é uma das principais causas que levam a diminuição da flexibilidade, causando algumas vezes alterações posturais ${ }^{14}$. Os benefícios do treinamento de flexibilidade são encontrados em diversas áreas, entre elas: prevenção de lesões, alívio da dor, relaxamento muscular e mental, melhora da amplitude de movimento (ADM) e postura ${ }^{15}$. O alongamento muscular é um importante componente de um programa de condicionamento físico, pois permite que o músculo recupere seu comprimento necessário para manter um ali- nhamento postural correto e a estabilidade articular, garantindo principalmente a integridade e a função muscular ${ }^{1}$. Vários métodos de alongamento têm sido descritos na literatura, incluindo alongamento estático, balístico e a facilitação neuromuscular proprioceptiva (FNP) $)^{15,16,17}$.

Assim como tem sido reconhecido que a atividade física regular traz efeitos saudáveis aos praticantes ${ }^{18}$, existe o conceito de reversibilidade, mostrando que, quando o treinamento físico é suspenso ou reduzido, os sistemas corporais se reajustam de acordo com a diminuição do estímulo ${ }^{19}$. O destreinamento físico resulta em perdas das adaptações do sistema cardiovascular e metabólicas do músculo esquelético. Em relação ao acompanhamento da qualidade de vida não existem muitos estudos relacionados a ela. Apenas Richards e $\mathrm{Scott}^{20}$ realizaram um estudo para avaliar os benefícios do exercício físico na fibromialgia e observaram manutenção da melhora da qualidade de vida após um ano. Atualmente, pesquisas têm sido realizadas para comprovar a relação dos exercícios físicos com a qualidade de vida. Entretanto, não comprovam os benefícios trazidos à população em geral (que não possui nenhum tipo de doença) e também não os acompanham ao longo do tempo. Portanto, o objetivo neste estudo foi analisar o impacto do alongamento muscular na qualidade de vida e na flexibilidade de mulheres jovens antes, imediatamente após e um ano após a sua realização.

\section{Material e métodos}

\section{Participantes}

Todas as alunas do $1^{\circ}$ ao $6^{\circ}$ semestre do curso de Fisioterapia de uma instituição de ensino superior da cidade de Osasco, com idade entre 17 e 25 anos, sedentárias, que tinham como atividade principal o estudo, responderam ao questionário de qualidade de vida SF-36. Os critérios de exclusão foram realizar outra atividade, como trabalhar ou praticar exercícios físicos, e possuir algum tipo de doença crônica, 
fatores esses que poderiam interferir na análise dos questionários. Das componentes da amostra que responderam ao SF-36, foram selecionadas as que obtiveram os 15 menores escores de qualidade de vida. Durante o estudo, houve a desistência de quatro participantes, sendo a amostra final constituída por 11 voluntárias.

\section{Procedimentos}

Após a seleção, as participantes receberam e assinaram um termo de consentimento livre e esclarecido, concordando com os termos da pesquisa, sendo o estudo conduzido de acordo com a Resolução 196/96 do Conselho Nacional de Saúde. Todos os procedimentos foram realizados no Laboratório de Fisioterapia da instituição. Na avaliação, além da aplicação do SF-36, foi medida a distância terceiro dedosolo com fita métrica, considerada como medida de flexibilidade ${ }^{21}$.

Antes do início de cada sessão de alongamento, realizou-se um breve aquecimento de cinco minutos, que consistia em caminhada numa pista de atletismo. O protocolo baseou-se no alongamento dos seguintes músculos bilateralmente: flexores dos dedos, flexor ulnar e radial do carpo, extensor longo e curto radial do carpo, extensor ulnar do carpo, tríceps braquial, esternocleidomastoideo, trapézio, romboide, peitoral maior, latíssimo do dorso, eretores da espinha, oblíquo externo e interno, reto femoral, adutores do quadril, glúteo máximo, gastrocnêmio, isquiotibiais e tibial anterior ${ }^{22}$. Foi realizado durante quatro semanas, com a frequência de três vezes por semana e cada sessão tinha a duração média de 40 minutos. Cada autoalongamento foi mantido por 30 segundos com mesmo tempo de descanso.

Imediatamente após e um ano após o período de alongamento, a medida da distância terceiro dedo-solo e o SF-36 foram reaplicados. Além disso, na última reavaliação, as participantes responderam a ficha de acompanhamento com intuito de verificar a prática de atividade física no último ano.

\section{Anólise estatística}

Toda a análise estatística foi realizada em nível de 5\% de significância. Os dados do SF-36 e a medida da distância terceiro dedo-solo foram analisados antes, imediatamente após e um ano após o período de alongamento por meio da análise de variância (ANOVA) de Friedman. No SF-36, cada aspecto (capacidade funcional, grau de dor, aspecto físico, estado geral de saúde, vitalidade, aspectos sociais, aspecto emocional e saúde mental) foi analisado separadamente. Já a análise da questão dois do SF-36 e da ficha de acompanhamento foi efetuada de forma descritiva, em porcentagens.

\section{Resultodos}

Os dados demográficos da amostra estudada podem ser observados na Tabela 1. Para o SF-36, os resultados demonstraram que não houve diferença estatisticamente significante na capacidade funcional $(p=0,20)$ e no estado geral de saúde $(p=0,08)$. Em todos os outros aspectos do SF-36, foram demonstradas diferenças significativas, sendo eles: aspecto físico $(p=0,01)$, grau de dor $(\mathrm{p}=0,01)$, vitalidade $(\mathrm{p}=0,01)$, aspectos sociais $(\mathrm{p}=0,01)$, aspecto emocional $(\mathrm{p}=0,01)$ e saúde mental $(p=0,01)$. Os valores médios antes, imediatamente após e um ano depois do período de alongamento podem ser observados na Tabela 2 .

Tabela 1: Médias e desvios-padrão (DP) da idade, estatura e massa das participantes $(\mathrm{n}=1 \mathrm{l})$

\begin{tabular}{c|c}
\hline Dados demográficos & Média \pm DP \\
\hline Idade (anos) & $20,00 \pm 1,00$ \\
\hline Estatura $(\mathrm{m})$ & $1,64 \pm 0,07$ \\
\hline Massa $(\mathrm{Kg})$ & $59,00 \pm 9,98$ \\
\hline
\end{tabular}

A questão dois do SF-36 é um item que não entra na pontuação e refere-se às alterações de saúde ocorridas no período de um ano. A Figura 1 ilustra os resultados dessa questão antes, imediatamente após e um ano após o período de alongamento. 
Tabela 2: Médias e desvios-padrão (DP) dos valores dos aspectos do SF-36 antes, imediatamente após e um ano após o período de alongamento e valor de $\mathrm{p}(\mathrm{n}=1 \mathrm{l})$

\begin{tabular}{|c|c|c|c|c|}
\hline & Antes & $\begin{array}{l}\text { Imediata- } \\
\text { mente após }\end{array}$ & $\begin{array}{l}\text { Um ano } \\
\text { após }\end{array}$ & p \\
\hline $\begin{array}{c}\text { Capacidade } \\
\text { funcional }\end{array}$ & $\begin{array}{c}65,91 \pm \\
30,73\end{array}$ & $\begin{array}{c}82,73 \pm \\
8,48\end{array}$ & $\begin{array}{c}79,09 \pm \\
21,07\end{array}$ & 0,20 \\
\hline $\begin{array}{l}\text { Aspecto } \\
\text { físico }\end{array}$ & $\begin{array}{c}50,00 \pm \\
31,62\end{array}$ & $\begin{array}{c}75,00 \pm \\
27,39\end{array}$ & $\begin{array}{c}86,36 \pm \\
25,89\end{array}$ & $0,01 *$ \\
\hline Grau de dor & $\begin{array}{c}47,64 \pm \\
16,19\end{array}$ & $\begin{array}{c}68,55 \pm \\
15,28 \\
\end{array}$ & $\begin{array}{c}61,55 \pm \\
18,22 \\
\end{array}$ & $0,01^{*}$ \\
\hline $\begin{array}{c}\text { Estado geral } \\
\text { de saúde }\end{array}$ & $\begin{array}{c}53,46 \pm \\
16,42 \\
\end{array}$ & $\begin{array}{c}70,00 \pm \\
17,69\end{array}$ & $\begin{array}{c}64,09 \pm \\
20,28\end{array}$ & 0,08 \\
\hline Vitalidade & $\begin{array}{c}34,55 \pm \\
11,93\end{array}$ & $\begin{array}{c}54,55 \pm \\
15,73\end{array}$ & $\begin{array}{c}50,91 \pm \\
17,15\end{array}$ & $0,01^{*}$ \\
\hline $\begin{array}{l}\text { Aspectos } \\
\text { sociais }\end{array}$ & $\begin{array}{c}50,00 \pm \\
17,68\end{array}$ & $\begin{array}{c}78,41 \pm \\
16,86\end{array}$ & $\begin{array}{c}79,55 \pm \\
21,85\end{array}$ & $0,01^{*}$ \\
\hline $\begin{array}{l}\text { Aspecto } \\
\text { emocional }\end{array}$ & $\begin{array}{c}36,36 \pm \\
37,87\end{array}$ & $\begin{array}{c}72,73 \pm \\
29,13\end{array}$ & $\begin{array}{c}84,85 \pm \\
27,34\end{array}$ & $0,01 *$ \\
\hline Saúde mental & $\begin{array}{c}38,55 \pm \\
16,03\end{array}$ & $\begin{array}{c}65,09 \pm \\
16,89\end{array}$ & $\begin{array}{c}61,82 \pm \\
20,43\end{array}$ & $0,01^{*}$ \\
\hline
\end{tabular}

* Diferenças estatisticamente significantes $(p<0,05)$.

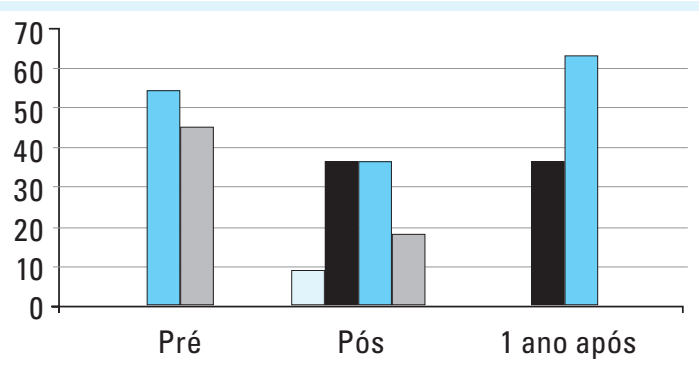
$\square$ - Muito melhor agora do que há um ano atrás;
- Um tanto melhor agora do que há um ano atrás;
$\square$ - Quase a mesma coisa do que há um ano atrás;
$\square$ - Um tanto pior agora do que há um ano atrás;
- Muito pior agora do que há um ano atrás.

Figura l: Valores, em porcentagem, das respostas das participantes em relação à questão dois do SF-36

Para a medida da distância terceiro dedosolo, os resultados demonstraram que ocorreu diferença estatisticamente significante $(p=0,02)$, quando comparados antes do alongamento, imediatamente após e o período de um ano de seguimento. Pela observação dos valores da medida da distância terceiro dedo-solo na Tabela 3, pode-se constatar que quase todas as participantes apresentaram uma melhora dessa medida no período pós-alongamento. Já, após um ano, observa-se que a maioria apresentou um aumento dos valores da medida da distância terceiro dedo-solo, e algumas retornaram à situação pré-alongamento. A Tabela 3 mostra os valores da distância terceiro dedo-solo para cada voluntário. Além disso, pela ficha de acompanhamento, observou-se que apenas $27,27 \%$ das participantes praticaram algum tipo de atividade física e o restante $(72,73 \%$ ) não realizou nenhum tipo de atividade.

Tabela 3: Valores, em centímetros, da medida da distôncia terceiro dedo-solo de cada participante antes, imediatamente após e um ano após o período de alongamento muscular $(\mathrm{n}=11)$

\begin{tabular}{c|c|c|c}
\hline Participante & Antes & $\begin{array}{c}\text { Imediata- } \\
\text { mente após }\end{array}$ & $\mathbf{1}$ ano após \\
\hline 1 & 15 & 12 & 15 \\
\hline 2 & -5 & -16 & -17 \\
\hline 3 & -5 & -12 & -5 \\
\hline 4 & -2 & -7 & -3 \\
\hline 5 & 12 & 9 & 15 \\
\hline 6 & 15 & 6 & 18 \\
\hline 7 & -18 & -2 & -3 \\
\hline 8 & 2 & -6 & 6 \\
\hline 9 & 11 & 4 & 6 \\
\hline 10 & 17 & 7 & -8 \\
\hline 11 & 22 & 7 & 14 \\
\hline
\end{tabular}

\section{Discussão}

Em relação à flexibilidade, os resultados da medida da distância terceiro dedo-solo mostraram que houve diferença significante antes, imediatamente após e um ano após o período de alongamento. Os valores dessa medida após um ano demonstraram que ocorreu uma diminuição da flexibilidade, o que pode ser explicado pelo longo período sem realizar o alongamen- 
to. Segundo Evangelista e Brum ${ }^{19}$, pessoas que treinam regularmente por um longo período de tempo parecem diferir daquelas que se exercitam apenas por alguns meses. Nos primeiros, um pequeno período de destreinamento físico não é suficiente para reverter uma adaptação de longa duração.

Driusso et al. ${ }^{23}$, em um estudo realizado em mulheres com osteoporose, verificaram que ocorreu melhora na flexibilidade, apresentando uma diferença média de $9 \mathrm{~cm}$ na medida da distância terceiro dedo-solo. Esses resultados são parecidos com os obtidos neste estudo imediatamente após o período de alongamento e apesar de o número de participantes na amostra ser semelhante, o tempo da sessão e a frequência do alongamento diferem do que foi utilizado nesta pesquisa, não permitindo assim maiores comparações.

Os questionários de qualidade de vida podem ser aplicados por meio de entrevista ou autoaplicação, na qual requerem menos tempo e permitem que o indivíduo responda no seu ritmo e possa voltar aos itens e refletir melhor sobre sua resposta. Neste estudo, utilizou-se a autoaplicação, pois a amostra avaliada inicialmente era composta por um grande número de participantes. Além disso, eram universitárias e, por conseguinte, possuíam um maior nível de entendimento em relação à população em geral. Optou-se pela utilização do SF-36 neste trabalho, porque é o questionário genérico de qualidade de vida mais utilizado, e por não ser específico para determinada população e/ou doença, possibilitou que a amostra de participantes saudáveis fosse avaliada.

Segundo Provout ${ }^{24}$, as conquistas tecnológicas e os avanços científicos tão necessários induzem o indivíduo a um estado de sedentarismo que, aliado a outros fatores, tais como estresse, alimentação inadequada, tabagismo e consumo excessivo de álcool, contribuem de maneira decisiva para o estado precário de saúde de grande parte da população. Ainda, os sujeitos sedentários podem ser acometidos por encurtamentos musculares e perda da flexibilidade, que acarretam algum tipo de dor e trazem limitações às atividades diárias realizadas. Isso consequentemente pode interferir na qualidade de vida, bem como na saúde e no estilo de vida, que estão diretamente relacionados a ela, o que também pode ter ocorrido com a amostra estudada.

Em estudo realizado por Dantas et al. ${ }^{3}$, verificou-se que, em $69,8 \%$ das pesquisas efetuadas no estado de São Paulo, investigaram-se pessoas portadoras de alguma patologia, entre elas: doenças pulmonares, renais, imunológicas, psiquiátricas, distúrbios gastrointestinais, problemas cardíacos e câncer. A reumatologia é uma área em que se realizam muitos trabalhos envolvendo qualidade de vida, e em que se estudam, além dessas mencionadas patologias, outras, tais como a fibromialgia ${ }^{1}$ e a osteoporo$\mathrm{se}^{23}$. Percebe-se, assim, a carência de pesquisas voltadas para a população em geral que, mesmo saudável, apresenta alterações na qualidade de vida.

Valim $^{25}$ estudou pacientes com fibromialgia e verificou que o condicionamento físico melhorou todos os aspectos avaliados pelo SF36 , enquanto que o de alongamento tornou melhor apenas dois, o aspecto emocional e a saúde mental. Em outro estudo, Valim et al. ${ }^{26}$ também observaram que os exercícios aeróbios foram superiores aos de alongamento nos aspectos emocionais e saúde mental do SF-36. Isso contraria os resultados obtidos neste estudo imediatamente após o período de alongamento, em que essa modalidade de exercício proporcionou melhora em quase todos os aspectos do SF-36, exceto na capacidade funcional e aspecto físico. Contudo, deve-se salientar que, neste trabalho, utilizou-se uma amostra de participantes saudáveis e os citados pacientes na pesquisa desses autores eram portadores de fibromialgia, que é uma doença com características próprias, tais como depressão e ansiedade. Em relação aos aspectos do SF36 que não apresentaram melhora (capacidade funcional e aspecto físico), isso pode ter ocorrido talvez porque em participantes considerados saudáveis essas alterações não são tão importantes como em acometidos de fibromialgia, não 
sendo possível observar melhora no resultado desses aspectos.

Além disso, estudos indicam que exercícios regulares têm promovido efeitos favoráveis na saúde mental, no bem-estar e na autoestima, e que alguns aspectos da qualidade de vida melhoram com tais atividades físicas ${ }^{27,28}$. Isso foi comprovado neste estudo, em que a realização de alongamento sugeria que apenas os domínios físicos da qualidade de vida obteriam alguma melhora, mas outros, como os emocionais, também melhoraram. Essa situação observada, segundo Godoy ${ }^{29}$, tem base em algumas hipóteses, entre elas a da interação social, que seria explicada a partir do sentimento de prazer da interação grupal e do reforço social recebido pelas pessoas que praticam o exercício físico. Tal hipótese pode comprovar a melhora ocorrida na amostra desta pesquisa, já que as sessões de alongamento foram realizadas em grupo.

Na literatura consultada, foi encontrado apenas um estudo que realizou acompanhamento da qualidade de vida. Richards e $\operatorname{Scott}^{20}$ observaram que os pacientes que realizaram exercícios aeróbios obtiveram uma melhora da qualidade de vida, que foi mantida por um ano. Esses resultados diferem dos encontrados neste trabalho, já que a maioria dos aspectos do SF-36 mostrou valores médios mais baixos no acompanhamento após um ano em relação ao imediatamente após, excetuando os aspectos físicos, emocionais e sociais. Entretanto, é importante ressaltar que a avaliação da qualidade de vida referida pelos autores foi realizada pelo Fibromyalgic Impact Questionnaire (FIQ), e os resultados do SF-36 após um ano não foram apresentados.

Ainda, pode-se observar pela análise da questão 2 do SF-36 que, no período após um ano, a maioria das participantes referiu que sua saúde estava quase a mesma coisa do que há um ano. Assim, sugere-se que os benefícios obtidos pelo alongamento muscular foram mantidos, porque a maioria dos valores dos aspectos não retornou aos valores pré-alongamento, apesar de terem diminuído. Por meio da ficha de acompanha- mento, observou-se que apenas $27,27 \%$ das participantes praticaram algum tipo de atividade física, como caminhada e condicionamento físico, sem regularidade, pois a prática variou de um a seis meses. Dessa forma, constata-se que o alongamento muscular, realizado pelo curto período de um mês, não incentivou as participantes para a prática regular de exercícios físicos.

A literatura é escassa em relação a estudos sobre qualidade de vida em pessoas saudáveis. Dentre os poucos trabalhos existentes, destaca-se o estudo de Macedo et al. ${ }^{30}$ em que avaliaram a qualidade de vida de 69 estudantes universitários por meio do SF-36, divididos em grupo de praticantes de exercício físico e grupo controle. Assim, realizaram uma comparação entre esses grupos e verificaram que ocorreu melhora em quase todos os aspectos da qualidade de vida, exceto no aspecto grau de dor. Esses resultados são semelhantes aos obtidos neste estudo, em que a maioria dos aspectos da qualidade de vida apresentou melhora significante, e também são encontrados em pesquisas que realizam o acompanhamento dos indivíduos por períodos determinados. Além disso, os estudos existentes de acompanhamento também são direcionados para populações que possuem algum tipo de doença.

As pessoas consideradas saudáveis são submetidas a fatores de risco que interferem na qualidade de vida, ocasionando sua piora. Em vista disso, a fisioterapia, por meio de métodos e técnicas, como, por exemplo, o alongamento muscular, seria importante para recuperar e também para manter a qualidade de vida e estaria assim possibilitando a promoção e prevenção da saúde da população. Entretanto, na literatura não há estudos que evidenciem essa alteração na qualidade de vida das pessoas em geral, sendo necessárias mais pesquisas sobre o tema.

\section{Conclusão}

Os dados desta pesquisa, nas condições experimentais utilizadas, permitem sugerir que 
a população considerada saudável, ao contrário do que é proposto, possui alterações na qualidade de vida. $\mathrm{O}$ alongamento muscular, como um recurso físico, ocasionou melhora nos componentes mental e social do SF-36, após sua realização, a qual foi mantida após um ano, pois apesar de ocorrer uma diminuição dos escores do SF-36, eles não retornaram aos valores de pré-alongamento. Em relação à flexibilidade, o alongamento muscular proporcionou melhora na amplitude de movimento da amostra estudada, a qual regrediu no período de um ano de inatividade física.

\section{Agradecimentos}

Os autores agradecem ao Programa Institucional de Bolsas de Iniciação Científica do Centro Universitário Fundação Instituto de Ensino para Osasco (UNIFIEO) pelo apoio financeiro para realização desta pesquisa.

\section{Referências}

1. Gashu BM, Marques AP, Ferreira EAG, Matsutani

LA. Eficácia da estimulação elétrica nervosa transcutânea (TENS) e dos exercícios de alongamento no alívio da dor e na melhora da qualidade de vida de pacientes com fibromialgia. Rev Fisioter Univ São Paulo. 2001;8(2):57-64.

2. Guyatt GH, Feeny Dh, Patrick DL. Measuring healthrelated quality of life. Ann Intern Med. 1993;118:622-9.

3. Dantas RAS, Sawada NO, Malerbo MB. Pesquisas sobre qualidade de vida: revisão da produção científica das universidades públicas do estado de São Paulo. Rev Lat Am Enfermagem. 2003;11(4):532-8.

4. Ferraz MB, Atra E. Avaliação da qualidade de vida em reumatologia. Rev Bras Reumatol. 1990;30(6):169-72.

5. Rodrigues Neto JF, Ferreira CG. Qualidade de vida como medida de desfecho em saúde. Rev Med Minas Gerais. 2003;13(1):42-6.
6. Ciconelli RM. Tradução para o português e validação do questionário genérico de avaliação da qualidade de vida: "Medical Outcomes Study 36-item shortform health survey (SF-36) [tese de doutorado]. São Paulo (SP): UNIFESP/EPM; 1997.

7. Ware J, Gandek B. Overview of the SF-36 health survey and the International Quality of Life Assessment (IQOLA) Project. J Clin Epidemiol. 1998;51(11):903-12.

8. Arnold LM, Witzeman KA, Swank ML, Mcelroy SL, Keck Jr PE. Health-related quality of life using the SF-36 in patients with bipolar disorder compared with patients with chronic back pain and the general population. J Affect Disord. 2000;57(1-3):235-9.

9. Brazier J, Usherwood T, Harper R, Thomas K. Deriving a preference-based single index from the UK SF-36 health survey. J Clin Epidemiol. 1998;51(11):1115-28.

10. Wiles NJ, Scott DGI, Barret EM, Merry P, Arie E, Gaffney K et al. Benchmarking: the five year outcome of rheumatoid arthritis assessed using a pain score, the Health Assessment Questionnaire, and the Short Form-36 (SF-36) in a community and a clinic based sample. Ann Rheum Dis. 2001;60(9):956-61.

11. Pellegrinotti IL. Atividade física e esporte: a importância no contexto saúde do ser humano. Rev Bras Ativ Fis Saúde. 1998;3(1):22-8.

12. Elley CR, Kerse N, Arroll B, Robinson E. Effectiveness of counselling patients on physical activity in general practice: cluster randomised controlled trial. BMJ. 2003;326:793-6.

13. Buckworth J, Nigg C. Physical activity, exercise, and sedentary behavior in college students. J Am Coll Health. 2004;53(1):28-34.

14. Pinfildi CE, Prado RP, Liebano RE. Efeito do alongamento estático após diatermia de ondas curtas versus alongamento estático nos músculos isquiotibiais em mulheres sedentárias. Fisioter Bras. 2004;5(2):119-24.

15. Lardner R. Stretching and flexibility: its importance in rehabilitation. J Body W Mov Ther. 2001;5(4):254-63.

16. Bandy WD, Irion JM, Briggler M. The effect of time and frequency of static stretching on flexibility of the hamstrings muscles. Phys Ther. 1997;77(10):1090-6.

17. Winters MV, Blake CG, Trost JS, Marcello-Brinker TB, Lowe L, Garber MB et al. Passive versus active stretching of hip flexor muscles in subjects with limited hip extension: a randomized clinical trial. Phys Ther. 2004;84(9):800-7. 
18. Palma A. Atividade física, processo saúde-doença e condições sócio-econômicas: uma revisão de literatura. Rev Paul Educ Fís. 2000;14(1):97-106.

19. Evangelista FS, Brum PC. Efeitos do destreinamento físico sobre a "performance" do atleta: uma revisão das alterações cardiovasculares e musculoesqueléticas. Rev Paul Educ Fís. 1999;13(2):239-49.

20. Richards SCM, Scott DL. Prescribed exercise in people with fibromyalgia: parallel group randomized controlled trial. BMJ. 2002;325(185):1-4.

21. Nonaka L, Caromano FA, Mendes FAS. Avaliação de dois testes de flexibilidade em idosos do sexo feminino - método fotográfico. Rev Terapia Ocupacional da Univ São Paulo. 1999;10(2/3):75-80.

22. Kisner C, Colby L. Exercícios terapêuticos, fundamentos e técnicas. São Paulo: Manole; 2004.

23. Driusso P, Oishi J, Rennó ACM, Ferreira V. Efeitos de um programa de atividade física na qualidade de vida de mulheres com osteoporose. Rev Fisioter Univ São Paulo. 2000;7(1/2):1-9.

24. Provout PA. A atividade física de todo dia... recomendações e cuidados. Rev Inst Ciênc Saúde. 1999;17(2):115-7.
25. Valim V. Estudos dos efeitos do condicionamento aeróbico e do alongamento na fibromialgia [tese de doutorado]. São Paulo (SP): UNIFESP/EPM; 2001.

26. Valim V, Oliveira L, Suda A, Silva L, Assis M, Neto $\mathrm{TB}$ et al. Aerobic fitness effects in fibromyalgia. J Rheumatol. 2003;30(5):1060-9.

27. Parente D. Influence of aerobic and stretching exercise on anxiety and sensation-seeking mood state. Percept Mot Skills. 2000; 90(1):347-8.

28. BagØien TE, Halvari H. Autonomous motivation: involvement in physical activity, and perceived sport competence: structural and mediator models. Percept Mot Skills. 2005;100:3-21.

29. Godoy RF. Benefícios do exercício físico sobre a área emocional. Movimento. 2002;8(2):7-16.

30. Macedo CSG, Garavello JJ, Oku EC, Miyagusuku FH, Agnoll PD, Nocetti PM. Benefícios do exercício físico para a qualidade de vida. Rev Bras Ativ Fís Saúde. 2003;8(2):19-27. 